\title{
Fine Structure Analysis of the Configuration System of V II. Part II: Odd-Parity Levels
}

\author{
Safa Bouazza1, Richard A. Holt², David S. Rosner², Nathan M. R. Armstrong3 \\ ${ }^{1}$ LISM, Université de Champagne-Ardenne, Reims, France \\ ${ }^{2}$ Department of Physics and Astronomy, University of Western Ontario, London, Canada \\ ${ }^{3}$ Department of Physics and Astronomy, Mc Master University, Hamilton, Canada \\ Email: safa.bouazza@univ-reims.fr
}

Received 29 November 2013; revised 3 January 2014; accepted 23 January 2014

Copyright (C) 2014 by authors and Scientific Research Publishing Inc.

This work is licensed under the Creative Commons Attribution International License (CC BY).

http://creativecommons.org/licenses/by/4.0/

(c) (i) Open Access

\begin{abstract}
The $3 d^{3} 4 p, 3 d^{3} 5 p$ and $3 d^{2} 4 s 4 p$ odd configurations of the V II spectrum have been reanalysed and three $3 d^{2} 4 s 4 p$ triplets are assigned higher energies than previously proposed. We have determined the fine structure parameters, the largest and next largest eigenvector percentages of levels, their calculated Landé gJ-factors and predicted positions for missing experimental levels up to $100,000 \mathrm{~cm}^{-1}$ for the $3 d^{2} 4 s 4 p$ configuration. Furthermore for the first time a hyperfine structure (HFS) parametric treatment, involving levels of these two configurations has been carried out. The deduced single-electron HFS parameter values are successfully checked with those obtained by means of $a b$ initio calculations.
\end{abstract}

\section{Keywords}

Fine Structure, Hyperfine Structure, Energy Levels, Ab Initio Calculations, V II Spectrum

\section{Introduction}

The first analysis of the V II spectrum was done by Meggers and Moore [1] who found most of the predicted terms of the $3 d^{3} 4 p$ configuration and several low terms of the $3 d^{2} 4 s 4 p$ configuration. Some years later this spectrum was reobserved by the Madrid Group [2] [3] who extended these observations to the vacuum ultraviolet and infrared regions. This permitted to revise the previous assignments and to establish new levels. This $\mathrm{V}^{+}$ion fine structure (fs) analysis continued to be improved with the passing years [4]-[7]. Recently we carried out fs studies of some singly ionised atoms, e.g. Ta II [8] or Nb II [9], in an effort to complete previous works and to eliminate erroneous level assignments. This time we propose a similar work concerning V II, which presents high interest for astrophysical investigations since it is very useful in the study of the history of nucleosynthesis, chemically peculiar stars and the sun. 


\section{Fine and Hyperfine Structure Analysis}

As mentioned in our past work, the complex configuration-interaction between the odd-parity levels, especially between $3 d 4 s^{2} 4 p, 3 d^{2} 4 s 4 p$ and $3 d^{3} 4 p$ of V II, would make this work difficult. To overcome this, we use the extensively tested method of [10]-[12] that is suitable for systems of many mutually interacting Rydberg configurations, with the fs Hamiltonian of [13]-[17]. For this work on V II, we used the following as the configuration basis set: $3 d 4 s^{2} 4 p, 3 d 4 s^{2} 5 p, 3 d^{2} 4 s 4 p, 3 d^{2} 4 s 5 p, 3 d^{3} 4 p, 3 d^{3} 5 p$. Since there are many interaction integrals in this basis, by constraining the radial integrals to physically reasonable ratios [10], which also required a few assumptions from Hartree-Fock calculations, the fit procedure became manageable. Due to the relatively low atomic number of vanadium, LS coupling is preferably used. We fit the odd parity levels, up to $88 \times 10^{3} \mathrm{~cm}^{-1}$, that have been observed experimentally [7]. The configurations $3 d^{2} 4 s 4 p$ and $3 d^{3} 4 p$ have their entire fs parameter set adjusted, while the majority of $3 d^{3} 5 p$ is adjusted. As the levels of the other three configurations are not yet observed experimentally, the fs parameters of states in $3 d 4 s^{2} 4 p$, $3 d 4 s^{2} 5 p$ and $3 d^{2} 4 s 5 p$, that are expected to perturb the states of the three lowest configurations, could not be fit efficiently. Therefore, all parameters in the configurations $3 d 4 s^{2} 4 p, 3 d 4 s^{2} 5 p$ and $3 d^{2} 4 s 5 p$ are fixed to the weighted values from ab intio calculations. Our fs least squares fit of 186 energy levels from [7] used 27 free parameters, for a total of 128 parameters. This fit accurately reproduced the experimental data, with a standard deviation of $70 \mathrm{~cm}^{-1}$, with the exception of three triplets in the $3 d^{2} 4 s 4 p$ configuration: ${ }^{3} F,{ }^{1} \mathrm{P} ;{ }^{3} \mathrm{D},{ }^{3} \mathrm{~F},{ }^{1} \mathrm{P} ;{ }^{3} \mathrm{~F}$ and ${ }^{3} \mathrm{~F},{ }^{1} \mathrm{P} ;{ }^{3} \mathrm{G}$ whose level positions should be located higher than given in [7].

In Table 1, we show the observed and calculated energy levels, percentages of leading eigenvector components with their LS-term symbols, along with observed and calculated $g_{\mathrm{J}}$-factors. The fitted fs parameters are listed in Table 2 and Table 3 along with, from the Cowan calculations [18], their weighted $a b$ initio values. The Slater integral ratio, $0.7959=\frac{F^{2}(3 d, 3 d)(f s)}{F^{2}(3 d, 3 d)(a b-\text { initio })}=\frac{62803}{78906}$, determined as in [8] [9], for the $3 d^{2} 4 s 4 p$ configuration, provides the weighting for all parameters except spin-orbit parameters $\zeta_{n d}$ and $\zeta_{n p}$. Not listed in Table 2 and Table 3 are those fs parameters which are fixed to zero as they are theoretically expected to be small. In Table 4 we propose our predicted positions for the energy levels of these three erroneous triplets. We furthermore present in Table 4 , for the $3 d^{2} 4 s 4 p$ configuration, predicted energy level values for all missing experimental values up to $100,000 \mathrm{~cm}^{-1}$ to suggest further experimental investigations.

A many-body parametrisation calculation for the HFS analysis allows us to exploit the similarities between configuration interaction effects seen in spin-orbit and hyperfine splitting. Using Equations (4) and (5) in [19] for the A and B HFS constants, the radial parameters $a_{n l}^{\kappa k}, b_{n l}^{\kappa k}, a_{i}$ and $b_{i}$ are determined by fits to experimental values.

The first published magnetic dipole HFS A constants of ${ }^{51} \mathrm{~V}$ II, consisting of 24 even levels and 31 odd levels, are determined in 2011 by Armstrong, Rosner and Holt applying fast-ion-beam laser-fluorescence spectroscopy [20], which we use for our HFS fit. Our HFS fit does not require us to make extra assumptions for the parameter values as the number of HFS A-values makes the fit overdetermined. We can see from the well-known equation

$$
a_{n l}^{\kappa k}=2 \mu_{0} \mu_{B} \mu_{I}\left\langle r^{-3}\right\rangle_{n l}^{\kappa k} / 4 \pi I=95.4128 g_{I}\left\langle r^{-3}\right\rangle_{n l}^{\kappa k}
$$

(in $\mathrm{MHz}$ ) that our values for $p$ - and d-electron HFS many-body parameters are valid; where we use $g_{I}=\mu_{I} / I=5.1485 / 3.5=1.471$ for ${ }^{51} \mathrm{~V}$ and show the computed expectation values $\left\langle r^{-3}\right\rangle_{n l}^{\kappa k}$ in Table 5.

Previously, it was found [9], even more so for p-electrons, that weighting the parameters $a_{n l}^{\kappa k}$ by a ratio of spin-orbit constants obtained thanks to fine structure study and $a b$ initio calculations, i.e. to multiply the second member of Equation (1) by: $\zeta_{n l}(f s) / \zeta_{n l}(a b$ initio) improves the agreement of the calculated HFS values to the experimental values. For d-electrons this ratio is generally close to one and is sometimes superfluous to insert it in (1).

To check the value of the most influential HFS-deduced parameter

$$
a_{4 s}^{10}=95.4218 g_{I}\left\langle r^{-3}\right\rangle_{4 s}^{10}=63.6145 g_{I} 4 \pi\left|\psi(0)^{2}\right|
$$

for open s-shell configurations which shows that $a_{4 s}^{10}$ is directly proportional to s-electron density $4 \pi\left|\psi(0)^{2}\right|$ 
Table 1. Comparison between the observed and calculated energy levels $\left(\mathrm{in} \cdot \mathrm{cm}^{-1}\right)$ and $\mathrm{g}_{\mathrm{J}}$-factors. For each state the parent terms are given immediately after the configuration label in columns $3 \& 4$.

\begin{tabular}{|c|c|c|c|c|c|}
\hline $\begin{array}{l}\text { Obs. energy } \\
\text { level [7] }\end{array}$ & $\begin{array}{l}\text { Calc. } \\
\text { eigenvalue }\end{array}$ & $\begin{array}{l}\text { Largest eigenvector } \\
\text { component (\%) }\end{array}$ & $\begin{array}{c}\text { Next largest eigenvector } \\
\text { component }\end{array}$ & $\begin{array}{l}\text { Obs } g_{J} \\
{[1]}\end{array}$ & Calc. g \\
\hline \multicolumn{6}{|l|}{$\mathbf{J}=\mathbf{0}$} \\
\hline 37201.35 & 37253.45 & $97.68 \mathrm{C}{ }^{4} \mathrm{~F} ;{ }^{5} \mathrm{D}$ & $1.34 \mathrm{C}{ }^{4} \mathrm{P} ;{ }^{5} \mathrm{D}$ & & 0.00 \\
\hline 46586.37 & 46620.65 & $43.78 \mathrm{C}{ }^{4} \mathrm{P} ;{ }^{5} \mathrm{D}$ & $43.23 \mathrm{C}{ }^{4} \mathrm{P} ;{ }^{3} \mathrm{P}$ & & 0.00 \\
\hline 47027.95 & 47042.90 & $55.67 \mathrm{C}{ }^{4} \mathrm{P} ;{ }^{5} \mathrm{D}$ & $32.80 \mathrm{C}{ }^{4} \mathrm{P} ;{ }^{3} \mathrm{P}$ & & 0.00 \\
\hline 48258.22 & 48424.73 & $90.02 \mathrm{C}^{2} \mathrm{P} ;{ }^{1} \mathrm{~S}$ & $6.83 \mathrm{C}{ }^{4} \mathrm{P} ;{ }^{3} \mathrm{P}$ & & 0.00 \\
\hline 50662.27 & 50595.02 & $66.11 \mathrm{C}{ }^{2} \mathrm{P} ;{ }^{3} \mathrm{P}$ & $25.90 \mathrm{C}{ }^{2} \mathrm{D} ;{ }^{3} \mathrm{P}$ & & 0.00 \\
\hline 54813.40 & 54818.13 & $40.26 \mathrm{C}^{2} \mathrm{D} ;{ }^{3} \mathrm{P}$ & $32.62 \mathrm{C}{ }^{2} \mathrm{P} ;{ }^{3} \mathrm{P}$ & & 0.00 \\
\hline 65781.80 & 65853.34 & $92.75 \mathrm{~B}{ }^{3} \mathrm{~F},{ }^{3} \mathrm{P} ;{ }^{5} \mathrm{D}$ & $4.95 \mathrm{~B}{ }^{3} \mathrm{P},{ }^{3} \mathrm{P} ;{ }^{5} \mathrm{D}$ & & 0.00 \\
\hline 75820.80 & 75761.45 & $88.60 \mathrm{~B}{ }^{3} \mathrm{P},{ }^{3} \mathrm{P} ;{ }^{1} \mathrm{~S}$ & $7.73 \mathrm{~B}{ }^{1} \mathrm{D},{ }^{3} \mathrm{P} ;{ }^{3} \mathrm{P}$ & & 0.00 \\
\hline 76521.80 & 76607.47 & $83.94 \mathrm{~B}{ }^{1} \mathrm{D},{ }^{3} \mathrm{P} ;{ }^{3} \mathrm{P}$ & $7.73 \mathrm{~B}{ }^{3} \mathrm{P},{ }^{3} \mathrm{P} ;{ }^{1} \mathrm{~S}$ & & 0.00 \\
\hline 78644.10 & 78617.11 & $61.15 \mathrm{C}^{2} \mathrm{D} ;{ }^{3} \mathrm{P}$ & $16.48 \mathrm{C}^{2} \mathrm{D} ;{ }^{3} \mathrm{P}$ & & 0.00 \\
\hline 84875.00 & 84904.22 & $83.55 \mathrm{~B}{ }^{3} \mathrm{P},{ }^{3} \mathrm{P} ;{ }^{3} \mathrm{P}$ & $9.62 \mathrm{C}^{2} \mathrm{D} ;{ }^{3} \mathrm{P}$ & & 0.00 \\
\hline \multicolumn{6}{|l|}{$\mathbf{J}=\mathbf{1}$} \\
\hline 36489.36 & 36443.81 & $62.48 \mathrm{C}{ }^{4} \mathrm{~F} ;{ }^{3} \mathrm{D}$ & $30.36 \mathrm{C}{ }^{4} \mathrm{~F} ;{ }^{5} \mathrm{~F}$ & 0.35 & 0.360 \\
\hline 36954.63 & 36926.18 & $68.43 \mathrm{C}{ }^{4} \mathrm{~F} ;{ }^{5} \mathrm{~F}$ & $26.99 \mathrm{C}{ }^{4} \mathrm{~F} ;{ }^{3} \mathrm{D}$ & 0.24 & 0.166 \\
\hline 37259.39 & 37307.41 & $94.94 \mathrm{C}{ }^{4} \mathrm{~F} ;{ }^{5} \mathrm{D}$ & $2.46 \mathrm{C}{ }^{4} \mathrm{P} ;{ }^{3} \mathrm{D}$ & 1.39 & 1.472 \\
\hline 46690.42 & 46707.50 & 49.36 C ${ }^{4} \mathrm{P} ;{ }^{5} \mathrm{D}$ & $40.43 \mathrm{C}{ }^{4} \mathrm{P} ;{ }^{3} \mathrm{P}$ & 1.44 & 1.518 \\
\hline 46754.59 & 46753.23 & 88.99 C ${ }^{4} \mathrm{P} ;{ }^{5} \mathrm{P}$ & $8.39 \mathrm{C}{ }^{4} \mathrm{P} ;{ }^{5} \mathrm{D}$ & 2.28 & 2.398 \\
\hline 47107.99 & 47103.52 & $43.39 \mathrm{C}{ }^{4} \mathrm{P} ;{ }^{3} \mathrm{P}$ & $39.12 \mathrm{C}{ }^{4} \mathrm{P} ;{ }^{5} \mathrm{D}$ & 1.43 & 1.583 \\
\hline 50473.78 & 50556.41 & $55.18 \mathrm{C}^{2} \mathrm{P} ;{ }^{3} \mathrm{D}$ & $23.64 \mathrm{C}{ }^{4} \mathrm{P} ;{ }^{3} \mathrm{D}$ & 0.49 & 0.601 \\
\hline 50738.76 & 50692.54 & $51.56 \mathrm{C}{ }^{2} \mathrm{P} ;{ }^{3} \mathrm{P}$ & $23.38 \mathrm{C}^{2} \mathrm{D} ;{ }^{3} \mathrm{P}$ & 1.39 & 1.401 \\
\hline 52181.18 & 52172.27 & $81.64 \mathrm{C}^{2} \mathrm{P} ;{ }^{3} \mathrm{~S}$ & $11.01 \mathrm{C}{ }^{4} \mathrm{P} ;{ }^{3} \mathrm{~S}$ & 1.85 & 1.973 \\
\hline 52604.20 & 52600.72 & $44.17 \mathrm{C}{ }^{4} \mathrm{P} ;{ }^{3} \mathrm{D}$ & $16.60 \mathrm{C}^{2} \mathrm{D} ;{ }^{1} \mathrm{P}$ & 0.63 & 0.655 \\
\hline 52803.75 & 52800.40 & $43.79 \mathrm{C}{ }^{2} \mathrm{D} ;{ }^{1} \mathrm{P}$ & $16.45 \mathrm{C}^{2} \mathrm{D} ;{ }^{1} \mathrm{P}$ & 0.92 & 0.876 \\
\hline 53751.48 & 53696.00 & $61.43 \mathrm{C}^{2} \mathrm{D} ;{ }^{3} \mathrm{D}$ & $16.94 \mathrm{C}^{2} \mathrm{D} ;{ }^{3} \mathrm{D}$ & 0.49 & 0.519 \\
\hline 54717.86 & 54729.40 & $37.13 \mathrm{C}^{2} \mathrm{D} ;{ }^{3} \mathrm{P}$ & $30.87 \mathrm{C}^{2} \mathrm{P} ;{ }^{3} \mathrm{P}$ & & 1.503 \\
\hline 55663.23 & 55811.34 & $71.62 \mathrm{C}{ }^{4} \mathrm{P} ;{ }^{3} \mathrm{~S}$ & $11.01 \mathrm{C}^{2} \mathrm{P} ;{ }^{3} \mathrm{~S}$ & 1.92 & 1.886 \\
\hline 56171.40 & 56056.95 & $74.74 \mathrm{C}{ }^{2} \mathrm{P} ;{ }^{1} \mathrm{P}$ & $9.93 \mathrm{C}^{4} \mathrm{P} ;{ }^{3} \mathrm{~S}$ & 1.05 & 1.097 \\
\hline 63549.20 & 63498.95 & $98.42 \mathrm{~B}{ }^{3} \mathrm{~F},{ }^{3} \mathrm{P} ;{ }^{5} \mathrm{~F}$ & $1.03 \mathrm{C}^{4} \mathrm{~F} ;{ }^{5} \mathrm{~F}$ & & 0.000 \\
\hline 64930.77 & 64888.59 & $86.02 \mathrm{C}^{2} \mathrm{~F} ;{ }^{3} \mathrm{D}$ & $5.48 \mathrm{~B}{ }^{3} \mathrm{~F},{ }^{3} \mathrm{P} ;{ }^{3} \mathrm{D}$ & 0.46 & 0.499 \\
\hline 65815.20 & 65889.67 & $92.39 \mathrm{~B}{ }^{3} \mathrm{~F},{ }^{3} \mathrm{P} ;{ }^{5} \mathrm{D}$ & $4.88 \mathrm{~B}{ }^{3} \mathrm{P},{ }^{3} \mathrm{P} ;{ }^{5} \mathrm{D}$ & & 1.496 \\
\hline 68759.20 & 68762.09 & $76.61 \mathrm{~B}{ }^{3} \mathrm{P},{ }^{3} \mathrm{P} ;{ }^{3} \mathrm{D}$ & $7.11 \mathrm{~B}{ }^{3} \mathrm{P},{ }^{3} \mathrm{P} ;{ }^{3} \mathrm{D}$ & & 0.502 \\
\hline 74723.00 & 74611.11 & $91.55 \mathrm{~B}{ }^{3} \mathrm{P},{ }^{3} \mathrm{P} ;{ }^{3} \mathrm{~S}$ & 2.82 C 2P; 3S & & 1.997 \\
\hline 75280.00 & 75235.89 & $92.68 \mathrm{~B}{ }^{3} \mathrm{P},{ }^{3} \mathrm{P} ;{ }^{5} \mathrm{D}$ & $5.21 \mathrm{~B}{ }^{2} \mathrm{~F} ;{ }^{3} \mathrm{P} ;{ }^{5} \mathrm{D}$ & & 1.497 \\
\hline
\end{tabular}




\section{Continued}

\begin{tabular}{|c|c|c|c|c|c|}
\hline 75716.00 & 75806.61 & $60.95 \mathrm{C}^{2} \mathrm{D} ;{ }^{3} \mathrm{D}$ & $16.80 \mathrm{C}^{2} \mathrm{D} ;{ }^{3} \mathrm{D}$ & & 0.504 \\
\hline 76454.70 & 76455.84 & $88.52 \mathrm{~B}{ }^{1} \mathrm{D},{ }^{3} \mathrm{P} ;{ }^{3} \mathrm{P}$ & $3.89 \mathrm{C}^{2} \mathrm{D} ;{ }^{3} \mathrm{P}$ & & 1.500 \\
\hline 77555.10 & 77752.08 & $69.62 \mathrm{~B}{ }^{1} \mathrm{D},{ }^{3} \mathrm{P} ;{ }^{3} \mathrm{D}$ & $7.42 \mathrm{C}{ }^{4} \mathrm{~F} ;{ }^{3} \mathrm{D}$ & & 0.611 \\
\hline 77853.60 & 77890.67 & $93.89 \mathrm{~B}{ }^{3} \mathrm{P},{ }^{3} \mathrm{P} ;{ }^{5} \mathrm{P}$ & $3.67 \mathrm{~B}{ }^{1} \mathrm{D},{ }^{3} \mathrm{P} ;{ }^{3} \mathrm{P}$ & & 2.401 \\
\hline 78569.10 & 78528.32 & $61.38 \mathrm{C}^{2} \mathrm{D} ;{ }^{3} \mathrm{P}$ & $16.51 \mathrm{C}^{2} \mathrm{D} ;{ }^{3} \mathrm{P}$ & & 1.493 \\
\hline 79259.09 & 79307.31 & $55.40 \mathrm{D}{ }^{4} \mathrm{~F} ;{ }^{3} \mathrm{D}$ & $20.23 \mathrm{D}{ }^{4} \mathrm{~F} ;{ }^{5} \mathrm{D}$ & & 0.635 \\
\hline 82366.50 & 82402.84 & $41.03 \mathrm{C}{ }^{2} \mathrm{D} ;{ }^{1} \mathrm{P}$ & 19.03 B ${ }^{3} \mathrm{P},{ }^{3} \mathrm{P} ;{ }^{1} \mathrm{P}$ & & 0.884 \\
\hline 82497.40 & 82679.91 & $56.98 \mathrm{~B}{ }^{3} \mathrm{P},{ }^{3} \mathrm{P} ;{ }^{3} \mathrm{D}$ & $13.51 \mathrm{C}{ }^{2} \mathrm{D} ;{ }^{1} \mathrm{P}$ & & 0.615 \\
\hline 84959.50 & 84975.66 & $84.08 \mathrm{~B}{ }^{3} \mathrm{P},{ }^{3} \mathrm{P} ;{ }^{3} \mathrm{P}$ & $9.38 \mathrm{C}^{2} \mathrm{D} ;{ }^{3} \mathrm{P}$ & & 1.501 \\
\hline \multicolumn{6}{|l|}{$\mathbf{J}=2$} \\
\hline 34592.75 & 34560.20 & $99.41 \mathrm{C}{ }^{4} \mathrm{~F} ;{ }^{5} \mathrm{G}$ & $0.22 \mathrm{D}{ }^{4} \mathrm{~F} ;{ }^{5} \mathrm{G}$ & 0.31 & 0.333 \\
\hline 36673.48 & 36639.07 & $60.47 \mathrm{C}{ }^{4} \mathrm{~F} ;{ }^{5} \mathrm{~F}$ & $31.55 \mathrm{C}{ }^{4} \mathrm{~F} ;{ }^{3} \mathrm{D}$ & 1.08 & 1.079 \\
\hline 37041.12 & 37011.05 & $52.59 \mathrm{C}{ }^{4} \mathrm{~F} ;{ }^{3} \mathrm{D}$ & $38.25 \mathrm{C}{ }^{4} \mathrm{~F} ;{ }^{5} \mathrm{~F}$ & 1.08 & 1.116 \\
\hline 37368.96 & 37409.91 & $89.41 \mathrm{C}{ }^{4} \mathrm{~F} ;{ }^{5} \mathrm{D}$ & $7.55 \mathrm{C}{ }^{4} \mathrm{~F} ;{ }^{3} \mathrm{D}$ & 1.39 & 1.473 \\
\hline 40001.70 & 39952.85 & $94.11 \mathrm{C}{ }^{4} \mathrm{~F} ;{ }^{3} \mathrm{~F}$ & $2.55 \mathrm{C}^{2} \mathrm{D} ;{ }^{3} \mathrm{~F}$ & 0.65 & 0.667 \\
\hline 46739.99 & 46745.10 & $63.02 \mathrm{C}{ }^{4} \mathrm{P} ;{ }^{3} \mathrm{P}$ & $17.22 \mathrm{C}{ }^{4} \mathrm{P} ;{ }^{5} \mathrm{D}$ & 1.48 & 1.521 \\
\hline 46879.88 & 46871.88 & $87.48 \mathrm{C}{ }^{4} \mathrm{P} ;{ }^{5} \mathrm{P}$ & $10.35 \mathrm{C}{ }^{4} \mathrm{P} ;{ }^{5} \mathrm{D}$ & 1.68 & 1.794 \\
\hline 47101.89 & 47101.19 & $69.16 \mathrm{C}{ }^{4} \mathrm{P} ;{ }^{5} \mathrm{D}$ & 19.27 C ${ }^{4} \mathrm{P} ;{ }^{3} \mathrm{P}$ & 1.47 & 1.519 \\
\hline 49201.64 & 49308.81 & $73.58 \mathrm{C}^{2} \mathrm{G} ;{ }^{3} \mathrm{~F}$ & $10.96 \mathrm{C}^{2} \mathrm{D} ;{ }^{3} \mathrm{~F}$ & 0.63 & 0.689 \\
\hline 49731.24 & 49798.88 & $95.20 \mathrm{C}{ }^{4} \mathrm{P} ;{ }^{5} \mathrm{~S}$ & $2.21 \mathrm{~B}{ }^{3} \mathrm{P},{ }^{3} \mathrm{P} ;{ }^{5} \mathrm{~S}$ & & 1.983 \\
\hline 49898.19 & 49856.76 & $47.05 \mathrm{C}^{2} \mathrm{P} ;{ }^{1} \mathrm{D}$ & $27.56 \mathrm{C}^{2} \mathrm{D} ;{ }^{1} \mathrm{D}$ & 0.93 & 1.000 \\
\hline 50775.47 & 50848.26 & $56.52 \mathrm{C}{ }^{2} \mathrm{P} ;{ }^{3} \mathrm{D}$ & $28.08 \mathrm{C}{ }^{4} \mathrm{P} ;{ }^{3} \mathrm{D}$ & 1.11 & 1.158 \\
\hline 51123.21 & 51070.76 & $55.95 \mathrm{C}{ }^{2} \mathrm{P} ;{ }^{3} \mathrm{P}$ & $28.13 \mathrm{C}{ }^{2} \mathrm{D} ;{ }^{3} \mathrm{P}$ & 1.51 & 1.496 \\
\hline 52245.69 & 52226.20 & $57.04 \mathrm{C}^{2} \mathrm{D} ;{ }^{3} \mathrm{~F}$ & 16.95 C ${ }^{2} \mathrm{D} ;{ }^{3} \mathrm{~F}$ & 0.68 & 0.692 \\
\hline 52700.05 & 52671.60 & $54.17 \mathrm{C}{ }^{4} \mathrm{P} ;{ }^{3} \mathrm{D}$ & $26.91 \mathrm{C}{ }^{2} \mathrm{P} ;{ }^{3} \mathrm{D}$ & 1.10 & 1.155 \\
\hline 53868.61 & 53822.45 & $64.72 \mathrm{C}^{2} \mathrm{D} ;{ }^{3} \mathrm{D}$ & 17.27 C ${ }^{2} \mathrm{D} ;{ }^{3} \mathrm{D}$ & 1.10 & 1.170 \\
\hline 54715.66 & 54707.65 & $37.25 \mathrm{C}{ }^{2} \mathrm{D} ;{ }^{3} \mathrm{P}$ & $35.10 \mathrm{C}{ }^{2} \mathrm{P} ;{ }^{3} \mathrm{P}$ & & 1.497 \\
\hline 57342.56 & 57227.51 & $40.78 \mathrm{C}{ }^{2} \mathrm{P} ;{ }^{1} \mathrm{D}$ & $39.75 \mathrm{C}{ }^{2} \mathrm{D} ;{ }^{1} \mathrm{D}$ & 0.98 & 1.002 \\
\hline 62084.94 & 62066.75 & $78.13 \mathrm{C}^{2} \mathrm{~F} ;{ }^{3} \mathrm{~F}$ & $9.36 \mathrm{~B}{ }^{3} \mathrm{~F},{ }^{3} \mathrm{P} ;{ }^{5} \mathrm{G}$ & 0.58 & 0.637 \\
\hline 62285.80 & 62299.79 & $89.81 \mathrm{~B}{ }^{3} \mathrm{~F},{ }^{3} \mathrm{P} ;{ }^{5} \mathrm{G}$ & $8.96 \mathrm{C}^{2} \mathrm{~F} ;{ }^{3} \mathrm{~F}$ & & 0.365 \\
\hline 63656.80 & 63610.68 & $98.33 \mathrm{~B}{ }^{3} \mathrm{~F},{ }^{3} \mathrm{P} ;{ }^{5} \mathrm{~F}$ & $1.02 \mathrm{C}{ }^{4} \mathrm{~F} ;{ }^{5} \mathrm{~F}$ & & 1.000 \\
\hline 64586.14 & 64567.59 & $77.95 \mathrm{C}^{2} \mathrm{~F} ;{ }^{1} \mathrm{D}$ & $11.49 \mathrm{C}{ }^{2} \mathrm{D} ;{ }^{1} \mathrm{D}$ & 1.03 & 1.000 \\
\hline 64804.12 & 64778.89 & $85.09 \mathrm{C}^{2} \mathrm{~F} ;{ }^{3} \mathrm{D}$ & $4.98 \mathrm{~B}{ }^{3} \mathrm{~F},{ }^{3} \mathrm{P} ;{ }^{3} \mathrm{D}$ & 1.02 & 1.165 \\
\hline 65884.40 & 65967.02 & $91.98 \mathrm{~B}{ }^{3} \mathrm{~F},{ }^{3} \mathrm{P} ;{ }^{5} \mathrm{D}$ & $4.78 \mathrm{~B}{ }^{3} \mathrm{P},{ }^{3} \mathrm{P} ;{ }^{5} \mathrm{D}$ & & 1.497 \\
\hline 67737.70 & 67723.47 & $79.30 \mathrm{~B}{ }^{3} \mathrm{~F},{ }^{3} \mathrm{P} ;{ }^{3} \mathrm{~F}$ & $8.74 \mathrm{C}^{2} \mathrm{~F} ;{ }^{3} \mathrm{~F}$ & & 0.668 \\
\hline 68797.40 & 68801.51 & $75.30 \mathrm{~B}{ }^{3} \mathrm{~F},{ }^{3} \mathrm{P} ;{ }^{3} \mathrm{D}$ & $6.89 \mathrm{~B}{ }^{3} \mathrm{P},{ }^{3} \mathrm{P} ;{ }^{3} \mathrm{D}$ & & 1.166 \\
\hline
\end{tabular}




\section{Continued}

\begin{tabular}{|c|c|c|c|c|c|}
\hline 70923.28 & 70933.46 & $78.84 \mathrm{~B}{ }^{3} \mathrm{~F},{ }^{3} \mathrm{P} ;{ }^{1} \mathrm{D}$ & $9.14 \mathrm{~B}{ }^{3} \mathrm{P},{ }^{3} \mathrm{P} ;{ }^{1} \mathrm{D}$ & & 1.002 \\
\hline 75401.00 & 75358.70 & $90.83 \mathrm{~B}{ }^{3} \mathrm{P},{ }^{3} \mathrm{P} ;{ }^{5} \mathrm{D}$ & $5.07 \mathrm{~B}{ }^{3} \mathrm{~F},{ }^{3} \mathrm{P} ;{ }^{5} \mathrm{D}$ & & 1.489 \\
\hline 75758.20 & 75826.19 & $57.76 \mathrm{C}^{2} \mathrm{D} ;{ }^{3} \mathrm{D}$ & $15.43 \mathrm{C}^{2} \mathrm{D} ;{ }^{3} \mathrm{D}$ & 1.14 & 1.147 \\
\hline 76220.10 & 76188.18 & $76.18 \mathrm{~B}{ }^{1} \mathrm{D},{ }^{3} \mathrm{P} ;{ }^{3} \mathrm{~F}$ & $6.37 \mathrm{~B}{ }^{3} \mathrm{~F},{ }^{3} \mathrm{P} ;{ }^{3} \mathrm{~F}$ & & 0.703 \\
\hline 76252.30 & 76283.18 & 88.72 $\mathrm{B}{ }^{1} \mathrm{D},{ }^{3} \mathrm{P} ;{ }^{3} \mathrm{P}$ & $3.65 \mathrm{C}^{2} \mathrm{D} ;{ }^{3} \mathrm{P}$ & & 1.498 \\
\hline 77603.40 & 77532.67 & $45.80 \mathrm{C}{ }^{2} \mathrm{D} ;{ }^{1} \mathrm{D}$ & $17.98 \mathrm{C}^{2} \mathrm{D} ;{ }^{1} \mathrm{D}$ & & 0.937 \\
\hline 77640.00 & 77775.38 & $29.65 \mathrm{~B}{ }^{1} \mathrm{D},{ }^{3} \mathrm{P} ;{ }^{3} \mathrm{D}$ & $20.85 \mathrm{~B}{ }^{3} \mathrm{P},{ }^{3} \mathrm{P} ;{ }^{5} \mathrm{P}$ & & 1.160 \\
\hline 77857.00 & 77853.90 & $31.78 \mathrm{C}^{2} \mathrm{D} ;{ }^{3} \mathrm{~F}$ & $22.75 \mathrm{~B}{ }^{1} \mathrm{D},{ }^{3} \mathrm{P} ;{ }^{3} \mathrm{D}$ & & 0.938 \\
\hline 78006.80 & 78076.34 & $70.62 \mathrm{~B}{ }^{3} \mathrm{P},{ }^{3} \mathrm{P} ;{ }^{5} \mathrm{P}$ & $21.31 \mathrm{~B}{ }^{1} \mathrm{D},{ }^{3} \mathrm{P} ;{ }^{3} \mathrm{D}$ & & 1.641 \\
\hline 78416.80 & 78361.81 & $61.83 \mathrm{C}^{2} \mathrm{D} ;{ }^{3} \mathrm{P}$ & $16.49 \mathrm{C}^{2} \mathrm{D} ;{ }^{3} \mathrm{P}$ & & 1.494 \\
\hline 79435.03 & 79492.88 & $61.76 \mathrm{D}{ }^{4} \mathrm{~F} ;{ }^{3} \mathrm{D}$ & $20.82 \mathrm{D}{ }^{4} \mathrm{~F} ;{ }^{5} \mathrm{D}$ & & 1.220 \\
\hline 79853.58 & 79943.33 & $85.50 \mathrm{D}{ }^{4} \mathrm{~F} ;{ }^{3} \mathrm{~F}$ & $4.55 \mathrm{~B}{ }^{3} \mathrm{~F},{ }^{1} \mathrm{P} ;{ }^{3} \mathrm{~F}$ & & 0.676 \\
\hline 82593.10 & 82687.44 & $75.31 \mathrm{~B}{ }^{3} \mathrm{P},{ }^{3} \mathrm{P} ;{ }^{3} \mathrm{D}$ & $9.14 \mathrm{~B}{ }^{1} \mathrm{D},{ }^{3} \mathrm{P} ;{ }^{3} \mathrm{D}$ & & 1.167 \\
\hline 85102.70 & 85094.79 & $76.29 \mathrm{~B}{ }^{3} \mathrm{P},{ }^{3} \mathrm{P} ;{ }^{3} \mathrm{P}$ & $8.08 \mathrm{C}^{2} \mathrm{D} ;{ }^{3} \mathrm{P}$ & & 1.450 \\
\hline 85632.80 & 85503.49 & $68.41 \mathrm{~B}{ }^{3} \mathrm{P},{ }^{3} \mathrm{P} ;{ }^{1} \mathrm{D}$ & $10.04 \mathrm{~B}{ }^{3} \mathrm{~F},{ }^{3} \mathrm{P} ;{ }^{1} \mathrm{D}$ & & 1.050 \\
\hline 88042.60 & 87898.97 & $87.08 \mathrm{~B}{ }^{1} \mathrm{G},{ }^{3} \mathrm{P} ;{ }^{3} \mathrm{~F}$ & $3.64 \mathrm{~B}{ }^{3} \mathrm{~F},{ }^{1} \mathrm{P} ;{ }^{3} \mathrm{~F}$ & & 0.666 \\
\hline \multicolumn{6}{|l|}{$\mathbf{J}=\mathbf{3}$} \\
\hline 34745.72 & 34708.47 & $99.48 \mathrm{C}{ }^{4} \mathrm{~F} ;{ }^{5} \mathrm{G}$ & $0.22 \mathrm{D}{ }^{4} \mathrm{~F} ;{ }^{5} \mathrm{G}$ & 0.93 & 0.917 \\
\hline 36919.21 & 36912.72 & $65.56 \mathrm{C}{ }^{4} \mathrm{~F} ;{ }^{5} \mathrm{~F}$ & $26.05 \mathrm{C}{ }^{4} \mathrm{~F} ;{ }^{3} \mathrm{D}$ & 1.24 & 1.287 \\
\hline 37204.98 & 37169.38 & $48.52 \mathrm{C}{ }^{4} \mathrm{~F} ;{ }^{3} \mathrm{D}$ & $33.15 \mathrm{C}{ }^{4} \mathrm{~F} ;{ }^{5} \mathrm{~F}$ & 1.32 & 1.327 \\
\hline 37520.57 & 37548.06 & $79.32 \mathrm{C}{ }^{4} \mathrm{~F} ;{ }^{5} \mathrm{D}$ & $16.95 \mathrm{C}{ }^{4} \mathrm{~F} ;{ }^{3} \mathrm{D}$ & 1.47 & 1.470 \\
\hline 39234.05 & 39281.20 & $91.32 \mathrm{C}{ }^{4} \mathrm{~F} ;{ }^{3} \mathrm{G}$ & $6.65 \mathrm{C}^{2} \mathrm{G} ;{ }^{3} \mathrm{G}$ & 0.84 & 0.752 \\
\hline 40195.52 & 40146.56 & $93.66 \mathrm{C}{ }^{4} \mathrm{~F} ;{ }^{3} \mathrm{~F}$ & $2.48 \mathrm{C}^{2} \mathrm{D} ;{ }^{3} \mathrm{~F}$ & 1.02 & 1.083 \\
\hline 47051.86 & 47030.91 & $95.85 \mathrm{C}{ }^{4} \mathrm{P} ;{ }^{5} \mathrm{P}$ & $3.57 \mathrm{C}{ }^{4} \mathrm{P} ;{ }^{5} \mathrm{D}$ & 1.55 & 1.662 \\
\hline 47181.18 & 47176.91 & $93.19 \mathrm{C}{ }^{4} \mathrm{P} ;{ }^{5} \mathrm{D}$ & $3.62 \mathrm{C}{ }^{4} \mathrm{P} ;{ }^{5} \mathrm{P}$ & 1.48 & 1.506 \\
\hline 48579.94 & 48604.39 & $81.79 \mathrm{C}^{2} \mathrm{G} ;{ }^{3} \mathrm{G}$ & $6.54 \mathrm{C}^{4} \mathrm{~F} ;{ }^{3} \mathrm{G}$ & 0.67 & 0.775 \\
\hline 49210.76 & 49255.07 & $40.64 \mathrm{C}^{2} \mathrm{G} ;{ }^{3} \mathrm{~F}$ & $36.78 \mathrm{C}^{2} \mathrm{G} ;{ }^{1} \mathrm{~F}$ & 0.99 & 1.019 \\
\hline 49568.42 & 49558.97 & $43.22 \mathrm{C}{ }^{2} \mathrm{G} ;{ }^{1} \mathrm{~F}$ & $39.20 \mathrm{C}^{2} \mathrm{G} ;{ }^{3} \mathrm{~F}$ & 0.97 & 1.040 \\
\hline 51085.71 & 51161.56 & $53.34 \mathrm{C}^{2} \mathrm{P} ;{ }^{3} \mathrm{D}$ & $33.43 \mathrm{C}{ }^{4} \mathrm{P} ;{ }^{3} \mathrm{D}$ & 1.27 & 1.327 \\
\hline 52391.94 & 52377.75 & $53.25 \mathrm{C}{ }^{2} \mathrm{D} ;{ }^{3} \mathrm{~F}$ & $15.52 \mathrm{C}^{2} \mathrm{D} ;{ }^{3} \mathrm{~F}$ & 1.07 & 1.118 \\
\hline 52767.30 & 52704.16 & $44.10 \mathrm{C}{ }^{4} \mathrm{P} ;{ }^{3} \mathrm{D}$ & $34.21 \mathrm{C}^{2} \mathrm{P} ;{ }^{3} \mathrm{D}$ & & 1.306 \\
\hline 53927.18 & 53891.67 & $68.76 \mathrm{C}^{2} \mathrm{D} ;{ }^{3} \mathrm{D}$ & $17.06 \mathrm{C}^{2} \mathrm{D} ;{ }^{3} \mathrm{D}$ & 1.37 & 1.325 \\
\hline 55141.98 & 55143.28 & $53.24 \mathrm{C}^{2} \mathrm{D} ;{ }^{1} \mathrm{~F}$ & $18.14 \mathrm{C}^{2} \mathrm{H} ;{ }^{3} \mathrm{G}$ & 0.94 & 0.959 \\
\hline 55349.58 & 55360.75 & $73.77 \mathrm{C}^{2} \mathrm{H} ;{ }^{3} \mathrm{G}$ & $12.59 \mathrm{C}^{2} \mathrm{D} ;{ }^{1} \mathrm{~F}$ & 0.82 & 0.801 \\
\hline 62133.30 & 62108.43 & $85.04 \mathrm{C}^{2} \mathrm{~F} ;{ }^{3} \mathrm{~F}$ & $8.62 \mathrm{~B}{ }^{3} \mathrm{~F},{ }^{3} \mathrm{P} ;{ }^{3} \mathrm{~F}$ & 1.00 & 1.079 \\
\hline 62444.10 & 62469.11 & $96.93 \mathrm{~B}{ }^{3} \mathrm{~F},{ }^{3} \mathrm{P} ;{ }^{5} \mathrm{G}$ & $2.31 \mathrm{C}^{2} \mathrm{~F} ;{ }^{3} \mathrm{~F}$ & & 0.921 \\
\hline
\end{tabular}




\section{Continued}

\begin{tabular}{|c|c|c|c|c|c|}
\hline 63816.70 & 63776.74 & $98.00 \mathrm{~B}{ }^{3} \mathrm{~F},{ }^{3} \mathrm{P} ;{ }^{5} \mathrm{~F}$ & $1.02 \mathrm{C}{ }^{4} \mathrm{~F} ;{ }^{5} \mathrm{~F}$ & & 1.249 \\
\hline 64057.54 & 63966.63 & $93.42 \mathrm{C}{ }^{2} \mathrm{~F} ;{ }^{3} \mathrm{G}$ & $2.78 \mathrm{C}^{2} \mathrm{H} ;{ }^{3} \mathrm{G}$ & 0.72 & 0.754 \\
\hline 64603.44 & 64597.08 & $86.12 \mathrm{C}{ }^{2} \mathrm{~F} ;{ }^{3} \mathrm{D}$ & $4.46 \mathrm{~B}^{3} \mathrm{~F},{ }^{3} \mathrm{P} ;{ }^{3} \mathrm{D}$ & 1.22 & 1.331 \\
\hline 65995.60 & 65962.16 & $91.53 \mathrm{~B}{ }^{3} \mathrm{~F},{ }^{3} \mathrm{P} ;{ }^{5} \mathrm{D}$ & $4.66 \mathrm{~B}{ }^{3} \mathrm{~F},{ }^{3} \mathrm{P} ;{ }^{5} \mathrm{D}$ & & 1.496 \\
\hline 66303.84 & 66090.73 & $72.05 \mathrm{C}^{2} \mathrm{~F} ;{ }^{1} \mathrm{~F}$ & $23.31 \mathrm{~B}{ }^{3} \mathrm{~F},{ }^{3} \mathrm{P} ;{ }^{1} \mathrm{~F}$ & 0.95 & 1.005 \\
\hline 67904.80 & 67897.11 & $78.91 \mathrm{~B}{ }^{3} \mathrm{~F},{ }^{3} \mathrm{P} ;{ }^{3} \mathrm{~F}$ & $8.38 \mathrm{C}^{2} \mathrm{~F} ;{ }^{3} \mathrm{~F}$ & & 1.084 \\
\hline 68945.00 & 68954.00 & $76.78 \mathrm{~B}{ }^{3} \mathrm{~F},{ }^{3} \mathrm{P} ;{ }^{3} \mathrm{D}$ & $7.08 \mathrm{~B}{ }^{3} \mathrm{P},{ }^{3} \mathrm{P} ;{ }^{3} \mathrm{D}$ & & 1.332 \\
\hline 69644.00 & 69611.72 & $91.59 \mathrm{~B}{ }^{3} \mathrm{~F},{ }^{3} \mathrm{P} ;{ }^{3} \mathrm{G}$ & $2.43 \mathrm{~B}{ }^{1} \mathrm{G},{ }^{3} \mathrm{P} ;{ }^{3} \mathrm{G}$ & & 0.755 \\
\hline 70936.01 & 71023.39 & $72.01 \mathrm{~B}{ }^{3} \mathrm{~F},{ }^{3} \mathrm{P} ;{ }^{1} \mathrm{~F}$ & 19.78 C ${ }^{2} \mathrm{~F} ;{ }^{1} \mathrm{~F}$ & & 0.999 \\
\hline 75573.90 & 75531.06 & $85.88 \mathrm{~B}{ }^{3} \mathrm{P},{ }^{3} \mathrm{P} ;{ }^{5} \mathrm{D}$ & $4.77 \mathrm{~B}{ }^{3} \mathrm{~F},{ }^{3} \mathrm{P} ;{ }^{5} \mathrm{D}$ & & 1.477 \\
\hline 75847.90 & 75897.82 & $59.41 \mathrm{C}^{2} \mathrm{D} ;{ }^{3} \mathrm{D}$ & $15.20 \mathrm{C}^{2} \mathrm{D} ;{ }^{3} \mathrm{D}$ & 1.27 & 1.334 \\
\hline 76385.40 & 76291.21 & $74.90 \mathrm{~B}{ }^{1} \mathrm{D},{ }^{3} \mathrm{P} ;{ }^{3} \mathrm{~F}$ & $5.96 \mathrm{~B}^{3} \mathrm{~F},{ }^{3} \mathrm{P} ;{ }^{3} \mathrm{~F}$ & & 1.108 \\
\hline 77686.00 & 77790.39 & $47.22 \mathrm{C}^{2} \mathrm{D} ;{ }^{3} \mathrm{~F}$ & $18.07 \mathrm{C}^{2} \mathrm{D} ;{ }^{3} \mathrm{~F}$ & & 1.155 \\
\hline 77841.90 & 77871.93 & $38.48 \mathrm{~B}{ }^{1} \mathrm{D},{ }^{3} \mathrm{P} ;{ }^{3} \mathrm{D}$ & $30.00 \mathrm{~B}{ }^{3} \mathrm{P},{ }^{3} \mathrm{P} ;{ }^{5} \mathrm{P}$ & & 1.384 \\
\hline 78301.00 & 78398.37 & $63.18 \mathrm{~B}{ }^{3} \mathrm{P},{ }^{3} \mathrm{P} ;{ }^{5} \mathrm{P}$ & $29.14 \mathrm{~B}{ }^{1} \mathrm{D},{ }^{3} \mathrm{P} ;{ }^{3} \mathrm{D}$ & & 1.546 \\
\hline 79327.56 & 79412.43 & $73.04 \mathrm{C}^{2} \mathrm{D} ;{ }^{1} \mathrm{~F}$ & $17.95 \mathrm{C}^{2} \mathrm{D} ;{ }^{1} \mathrm{~F}$ & & 1.002 \\
\hline 79696.98 & 79750.85 & $72.46 \mathrm{D}{ }^{4} \mathrm{~F} ;{ }^{3} \mathrm{D}$ & $12.77 \mathrm{D}{ }^{4} \mathrm{~F} ;{ }^{5} \mathrm{D}$ & & 1.347 \\
\hline 79881.11 & 80046.73 & $90.08 \mathrm{~B}{ }^{1} \mathrm{G},{ }^{3} \mathrm{P} ;{ }^{3} \mathrm{G}$ & $2.81 \mathrm{~B}{ }^{3} \mathrm{~F},{ }^{1} \mathrm{P} ;{ }^{3} \mathrm{G}$ & & 0.750 \\
\hline 80057.61 & 80122.35 & $61.69 \mathrm{D}^{4} \mathrm{~F} ;{ }^{3} \mathrm{~F}$ & $24.84 \mathrm{D}{ }^{4} \mathrm{~F} ;{ }^{3} \mathrm{G}$ & & 1.005 \\
\hline 80463.03 & 80253.33 & $70.86 \mathrm{D}{ }^{4} \mathrm{~F} ;{ }^{3} \mathrm{G}$ & $22.57 \mathrm{D}{ }^{4} \mathrm{~F} ;{ }^{3} \mathrm{~F}$ & & 0.838 \\
\hline 82715.10 & 82789.09 & $76.44 \mathrm{~B}{ }^{3} \mathrm{P},{ }^{3} \mathrm{P} ;{ }^{3} \mathrm{D}$ & $7.64 \mathrm{~B}{ }^{3} \mathrm{~F},{ }^{3} \mathrm{P} ;{ }^{3} \mathrm{D}$ & & 1.334 \\
\hline 87933.50 & 87806.42 & 88.89 B ${ }^{1} \mathrm{G},{ }^{3} \mathrm{P} ;{ }^{3} \mathrm{~F}$ & $3.22 \mathrm{~B}{ }^{1} \mathrm{D},{ }^{3} \mathrm{P} ;{ }^{3} \mathrm{~F}$ & & 1.083 \\
\hline \multicolumn{6}{|l|}{$\mathbf{J}=4$} \\
\hline 34946.55 & 34903.61 & $99.55 \mathrm{C}{ }^{4} \mathrm{~F} ;{ }^{5} \mathrm{G}$ & $0.22 \mathrm{D}{ }^{4} \mathrm{~F} ;{ }^{5} \mathrm{G}$ & 1.14 & 1.150 \\
\hline 37150.51 & 37166.50 & $97.75 \mathrm{C}{ }^{4} \mathrm{~F} ;{ }^{5} \mathrm{~F}$ & $0.99 \mathrm{~B}{ }^{3} \mathrm{~F},{ }^{3} \mathrm{P} ;{ }^{5} \mathrm{~F}$ & & 1.351 \\
\hline 37531.08 & 37596.15 & $96.63 \mathrm{C}^{4} \mathrm{~F} ;{ }^{5} \mathrm{D}$ & $1.27 \mathrm{C}{ }^{4} \mathrm{P} ;{ }^{5} \mathrm{D}$ & 1.44 & 1.499 \\
\hline 39403.74 & 39446.81 & $91.14 \mathrm{C}{ }^{4} \mathrm{~F} ;{ }^{3} \mathrm{G}$ & $6.53 \mathrm{C}^{2} \mathrm{G} ;{ }^{3} \mathrm{G}$ & 1.03 & 1.052 \\
\hline 40430.04 & 40384.04 & $93.74 \mathrm{C}{ }^{4} \mathrm{~F} ;{ }^{3} \mathrm{~F}$ & $2.39 \mathrm{C}^{2} \mathrm{D} ;{ }^{3} \mathrm{~F}$ & 1.22 & 1.250 \\
\hline 47056.32 & 47093.49 & $86.49 \mathrm{C}^{2} \mathrm{G} ;{ }^{3} \mathrm{H}$ & $12.64 \mathrm{C}^{2} \mathrm{H} ;{ }^{3} \mathrm{H}$ & 0.78 & 0.801 \\
\hline 47420.25 & 47399.36 & $97.20 \mathrm{C}{ }^{4} \mathrm{P} ;{ }^{5} \mathrm{D}$ & $1.35 \mathrm{~B}{ }^{3} \mathrm{~F},{ }^{3} \mathrm{P} ;{ }^{5} \mathrm{D}$ & 2.28 & 1.501 \\
\hline 48730.72 & 48758.93 & $87.68 \mathrm{C}{ }^{2} \mathrm{G} ;{ }^{3} \mathrm{G}$ & $6.70 \mathrm{C}{ }^{4} \mathrm{~F} ;{ }^{3} \mathrm{G}$ & 1.02 & 1.052 \\
\hline 49268.55 & 49340.63 & $62.64 \mathrm{C}^{2} \mathrm{G} ;{ }^{3} \mathrm{~F}$ & $25.11 \mathrm{C}^{2} \mathrm{G} ;{ }^{1} \mathrm{G}$ & 1.18 & 1.183 \\
\hline 49723.61 & 49759.63 & $71.93 \mathrm{C}{ }^{2} \mathrm{G} ;{ }^{1} \mathrm{G}$ & $20.84 \mathrm{C}{ }^{2} \mathrm{G} ;{ }^{3} \mathrm{~F}$ & 0.96 & 1.061 \\
\hline 52082.82 & 52091.71 & $85.42 \mathrm{C}^{2} \mathrm{H} ;{ }^{3} \mathrm{H}$ & $12.62 \mathrm{C}^{2} \mathrm{G} ;{ }^{3} \mathrm{H}$ & 0.70 & 0.803 \\
\hline 52657.47 & 52623.38 & $64.03 \mathrm{C}^{2} \mathrm{D} ;{ }^{3} \mathrm{~F}$ & $18.58 \mathrm{C}^{2} \mathrm{D} ;{ }^{3} \mathrm{~F}$ & 1.18 & 1.250 \\
\hline 54144.17 & 54126.35 & $82.69 \mathrm{C}^{2} \mathrm{H} ;{ }^{1} \mathrm{G}$ & $11.76 \mathrm{C}^{2} \mathrm{~F} ;{ }^{1} \mathrm{G}$ & 1.00 & 1.001 \\
\hline
\end{tabular}




\section{Continued}

\begin{tabular}{|c|c|c|c|c|c|}
\hline 55304.30 & 55337.50 & $89.90 \mathrm{C}^{2} \mathrm{H} ;{ }^{3} \mathrm{G}$ & $2.41 \mathrm{C}^{2} \mathrm{~F} ;{ }^{3} \mathrm{G}$ & 1.02 & 1.049 \\
\hline 62176.19 & 62155.68 & $87.41 \mathrm{C}^{2} \mathrm{~F} ;{ }^{3} \mathrm{~F}$ & $8.20 \mathrm{~B}{ }^{3} \mathrm{~F},{ }^{3} \mathrm{P} ;{ }^{3} \mathrm{~F}$ & 1.36 & 1.249 \\
\hline 62682.06 & 62706.56 & $98.85 \mathrm{~B}{ }^{3} \mathrm{~F},{ }^{3} \mathrm{P} ;{ }^{5} \mathrm{G}$ & $0.44 \mathrm{C}^{2} \mathrm{~F} ;{ }^{3} \mathrm{~F}$ & & 1.151 \\
\hline 64026.20 & 63991.68 & $89.51 \mathrm{~B}{ }^{3} \mathrm{~F},{ }^{3} \mathrm{P} ;{ }^{5} \mathrm{~F}$ & $8.38 \mathrm{C}^{2} \mathrm{~F} ;{ }^{3} \mathrm{G}$ & & 1.323 \\
\hline 64130.80 & 64051.47 & $84.92 \mathrm{C}^{2} \mathrm{~F} ;{ }^{3} \mathrm{G}$ & $8.92 \mathrm{~B}{ }^{3} \mathrm{~F},{ }^{3} \mathrm{P} ;{ }^{5} \mathrm{~F}$ & 1.02 & 1.078 \\
\hline 65790.18 & 65865.87 & $86.11 \mathrm{C}{ }^{2} \mathrm{~F} ;{ }^{1} \mathrm{G}$ & $11.49 \mathrm{C}{ }^{2} \mathrm{H} ;{ }^{1} \mathrm{G}$ & 0.94 & 1.000 \\
\hline 66157.60 & 66271.20 & $92.46 \mathrm{~B}{ }^{3} \mathrm{~F},{ }^{3} \mathrm{P} ;{ }^{5} \mathrm{D}$ & $4.63 \mathrm{~B}{ }^{3} \mathrm{P},{ }^{3} \mathrm{P} ;{ }^{5} \mathrm{D}$ & & 1.499 \\
\hline 68147.06 & 68134.81 & $80.34 \mathrm{~B}{ }^{3} \mathrm{~F},{ }^{3} \mathrm{P} ;{ }^{3} \mathrm{~F}$ & $7.95 \mathrm{C}^{2} \mathrm{~F} ;{ }^{3} \mathrm{~F}$ & & 1.251 \\
\hline 69911.80 & 69871.78 & $92.89 \mathrm{~B}{ }^{3} \mathrm{~F},{ }^{3} \mathrm{P} ;{ }^{3} \mathrm{G}$ & $2.55 \mathrm{~B}{ }^{1} \mathrm{G},{ }^{3} \mathrm{P} ;{ }^{3} \mathrm{G}$ & & 1.051 \\
\hline 75824.80 & 75757.49 & 82.72 $\mathrm{B}{ }^{3} \mathrm{P},{ }^{3} \mathrm{P} ;{ }^{5} \mathrm{D}$ & $9.13 \mathrm{~B}{ }^{1} \mathrm{D},{ }^{3} \mathrm{P} ;{ }^{3} \mathrm{~F}$ & & 1.473 \\
\hline 76643.30 & 76520.70 & $70.04 \mathrm{~B}{ }^{1} \mathrm{D},{ }^{3} \mathrm{P} ;{ }^{3} \mathrm{~F}$ & $10.45 \mathrm{~B}{ }^{3} \mathrm{P},{ }^{3} \mathrm{P} ;{ }^{5} \mathrm{D}$ & & 1.278 \\
\hline 77968.90 & 77925.51 & $58.96 \mathrm{C}^{2} \mathrm{D} ;{ }^{3} \mathrm{~F}$ & $22.39 \mathrm{C}^{2} \mathrm{D} ;{ }^{3} \mathrm{~F}$ & & 1.251 \\
\hline 80013.14 & 80086.00 & $91.91 \mathrm{~B}{ }^{1} \mathrm{G},{ }^{3} \mathrm{P} ;{ }^{3} \mathrm{G}$ & $2.50 \mathrm{~B}{ }^{3} \mathrm{~F},{ }^{3} \mathrm{P} ;{ }^{3} \mathrm{G}$ & & 1.050 \\
\hline 80298.06 & 80355.08 & $53.56 \mathrm{D}{ }^{4} \mathrm{~F} ;{ }^{3} \mathrm{~F}$ & $38.08 \mathrm{D}{ }^{4} \mathrm{~F} ;{ }^{3} \mathrm{G}$ & & 1.175 \\
\hline 80595.56 & 80486.10 & $59.16 \mathrm{D}{ }^{4} \mathrm{~F} ;{ }^{3} \mathrm{G}$ & $34.75 \mathrm{D}{ }^{4} \mathrm{~F} ;{ }^{3} \mathrm{~F}$ & & 1.129 \\
\hline 83962.90 & 83985.15 & $99.36 \mathrm{~B}{ }^{1} \mathrm{G},{ }^{3} \mathrm{P} ;{ }^{3} \mathrm{H}$ & $0.26 \mathrm{C}^{2} \mathrm{H} ;{ }^{3} \mathrm{H}$ & & 0.800 \\
\hline 87789.70 & 87674.31 & $89.42 \mathrm{~B}{ }^{1} \mathrm{G},{ }^{3} \mathrm{P} ;{ }^{3} \mathrm{~F}$ & $3.29 \mathrm{~B}{ }^{1} \mathrm{D},{ }^{3} \mathrm{P} ;{ }^{3} \mathrm{~F}$ & & 1.250 \\
\hline \multicolumn{6}{|l|}{$\mathbf{J}=\mathbf{5}$} \\
\hline 35193.13 & 35144.52 & $99.60 \mathrm{C}{ }^{4} \mathrm{~F} ;{ }^{5} \mathrm{G}$ & $0.22 \mathrm{D}{ }^{4} \mathrm{~F} ;{ }^{5} \mathrm{G}$ & 1.16 & 1.267 \\
\hline 37352.45 & 37375.04 & $97.92 \mathrm{C}{ }^{4} \mathrm{~F} ;{ }^{5} \mathrm{~F}$ & $0.99 \mathrm{~B}{ }^{3} \mathrm{~F},{ }^{3} \mathrm{P} ;{ }^{5} \mathrm{~F}$ & 1.40 & 1.399 \\
\hline 39612.96 & 39657.86 & $91.10 \mathrm{C}{ }^{4} \mathrm{~F} ;{ }^{3} \mathrm{G}$ & $6.44 \mathrm{C}^{2} \mathrm{G} ;{ }^{3} \mathrm{G}$ & 1.19 & 1.202 \\
\hline 47297.04 & 47322.97 & $85.79 \mathrm{C}^{2} \mathrm{G} ;{ }^{3} \mathrm{H}$ & $12.98 \mathrm{C}^{2} \mathrm{H} ;{ }^{3} \mathrm{H}$ & 1.01 & 1.034 \\
\hline 48853.04 & 48880.09 & $85.03 \mathrm{C}^{2} \mathrm{G} ;{ }^{3} \mathrm{G}$ & $6.75 \mathrm{C}{ }^{4} \mathrm{~F} ;{ }^{3} \mathrm{G}$ & 1.22 & 1.190 \\
\hline 49593.36 & 49581.65 & $72.03 \mathrm{C}^{2} \mathrm{G} ;{ }^{1} \mathrm{H}$ & $22.36 \mathrm{C}^{2} \mathrm{H} ;{ }^{1} \mathrm{H}$ & 0.95 & 1.008 \\
\hline 52153.46 & 52166.53 & $86.17 \mathrm{C}^{2} \mathrm{H} ;{ }^{3} \mathrm{H}$ & $12.99 \mathrm{C}^{2} \mathrm{G} ;{ }^{3} \mathrm{H}$ & 0.98 & 1.034 \\
\hline 52877.89 & 52870.87 & $98.95 \mathrm{C}^{2} \mathrm{H} ;{ }^{3} \mathrm{I}$ & $0.48 \mathrm{C}^{2} \mathrm{G} ;{ }^{1} \mathrm{H}$ & 0.84 & 0.835 \\
\hline 55206.79 & 55117.15 & $61.02 \mathrm{C}^{2} \mathrm{H} ;{ }^{3} \mathrm{G}$ & $25.31 \mathrm{C}^{2} \mathrm{H} ;{ }^{1} \mathrm{H}$ & 1.15 & 1.133 \\
\hline 55499.34 & 55390.59 & $49.51 \mathrm{C}^{2} \mathrm{H} ;{ }^{1} \mathrm{H}$ & $30.86 \mathrm{C}^{2} \mathrm{H} ;{ }^{3} \mathrm{G}$ & 1.03 & 1.067 \\
\hline 62987.60 & 63006.78 & $99.33 \mathrm{~B}{ }^{3} \mathrm{~F},{ }^{3} \mathrm{P} ;{ }^{5} \mathrm{G}$ & $0.38 \mathrm{~B}{ }^{3} \mathrm{~F},{ }^{3} \mathrm{P} ;{ }^{5} \mathrm{G}$ & & 1.267 \\
\hline 64229.19 & 64148.53 & $91.63 \mathrm{C}^{2} \mathrm{~F} ;{ }^{3} \mathrm{G}$ & $3.16 \mathrm{~B}{ }^{3} \mathrm{~F},{ }^{3} \mathrm{P} ;{ }^{5} \mathrm{~F}$ & & 1.207 \\
\hline 64286.60 & 64270.71 & $95.39 \mathrm{~B}{ }^{3} \mathrm{~F},{ }^{3} \mathrm{P} ;{ }^{5} \mathrm{~F}$ & $3.19 \mathrm{C}^{2} \mathrm{~F} ;{ }^{3} \mathrm{G}$ & & 1.394 \\
\hline 70227.60 & 70184.45 & $93.39 \mathrm{~B}{ }^{3} \mathrm{~F},{ }^{3} \mathrm{P} ;{ }^{3} \mathrm{G}$ & $2.63 \mathrm{~B}{ }^{1} \mathrm{G},{ }^{3} \mathrm{P} ;{ }^{3} \mathrm{G}$ & & 1.201 \\
\hline 80155.72 & 80137.63 & $92.40 \mathrm{~B}{ }^{1} \mathrm{G},{ }^{3} \mathrm{P} ;{ }^{3} \mathrm{G}$ & $2.60 \mathrm{~B}{ }^{3} \mathrm{~F},{ }^{3} \mathrm{P} ;{ }^{3} \mathrm{G}$ & & 1.200 \\
\hline 80767.64 & 80698.89 & $97.24 \mathrm{D}{ }^{4} \mathrm{~F} ;{ }^{3} \mathrm{G}$ & $0.61 \mathrm{~B}{ }^{3} \mathrm{~F},{ }^{1} \mathrm{P} ;{ }^{3} \mathrm{G}$ & & 1.201 \\
\hline 84137.30 & 84152.34 & 99.36 $\mathrm{B}{ }^{1} \mathrm{G},{ }^{3} \mathrm{P} ;{ }^{3} \mathrm{H}$ & $0.26 \mathrm{C}^{2} \mathrm{H} ;{ }^{3} \mathrm{H}$ & & 1.034 \\
\hline
\end{tabular}




\section{Continued}

\begin{tabular}{|c|c|c|c|c|c|}
\hline $\mathbf{J}=\mathbf{6}$ & & & & & \\
\hline 35483.39 & 35428.86 & $99.62 \mathrm{C}{ }^{4} \mathrm{~F} ;{ }^{5} \mathrm{G}$ & $0.22 \mathrm{D}{ }^{4} \mathrm{~F} ;{ }^{5} \mathrm{G}$ & & 1.334 \\
\hline 47607.79 & 47616.47 & $86.36 \mathrm{C}^{2} \mathrm{G} ;{ }^{3} \mathrm{H}$ & $13.19 \mathrm{C}^{2} \mathrm{H} ;{ }^{3} \mathrm{H}$ & 1.13 & 1.167 \\
\hline 52252.60 & 52272.52 & $85.96 \mathrm{C}^{2} \mathrm{H} ;{ }^{3} \mathrm{H}$ & 13.27 C ${ }^{2} \mathrm{G} ;{ }^{3} \mathrm{H}$ & 1.04 & 1.166 \\
\hline 53076.71 & 53067.02 & $99.45 \mathrm{C}^{2} \mathrm{H} ;{ }^{3} \mathrm{I}$ & $0.31 \mathrm{C}^{2} \mathrm{H} ;{ }^{3} \mathrm{H}$ & 0.98 & 1.024 \\
\hline 55403.30 & 55580.73 & $99.59 \mathrm{C}{ }^{2} \mathrm{H} ;{ }^{1} \mathrm{I}$ & $0.23 \mathrm{C}^{2} \mathrm{H} ;{ }^{3} \mathrm{H}$ & 1.01 & 1.001 \\
\hline 63357.30 & 63366.75 & $99.49 \mathrm{~B}{ }^{3} \mathrm{~F},{ }^{3} \mathrm{P} ;{ }^{5} \mathrm{G}$ & $0.38 \mathrm{~B}{ }^{3} \mathrm{~F},{ }^{3} \mathrm{P} ;{ }^{5} \mathrm{G}$ & & 1.334 \\
\hline 84342.90 & 84349.18 & 99.49 B ${ }^{1} \mathrm{G},{ }^{3} \mathrm{P} ;{ }^{3} \mathrm{H}$ & $0.26 \mathrm{C}^{2} \mathrm{H} ;{ }^{3} \mathrm{H}$ & & 1.167 \\
\hline \multicolumn{6}{|l|}{$\mathbf{J}=7$} \\
\hline 53319.56 & 53305.96 & $99.84 \mathrm{C}^{2} \mathrm{H} ;{ }^{3} \mathrm{I}$ & $0.15 \mathrm{D}^{2} \mathrm{H} ;{ }^{3} \mathrm{I}$ & & 1.143 \\
\hline
\end{tabular}

B: $3 d^{2} 4 s 4 p$ configuration; C: $3 d^{3} 4 p$ configuration; D: $3 d^{3} 5 p$ configuration.

Table 2. Fine structure fitted parameters values (in $\cdot \mathrm{cm}^{-1}$ ) for the odd-parity levels of V II (Fit) and corresponding weighted $a b$ initio values.

\begin{tabular}{cccccccc}
\hline Configuration & $3 d^{1} 4 s^{2} 4 p^{1}$ & \multicolumn{2}{c}{$3 d^{2} 4 s^{1} 4 p^{1}$} & & $3 d^{3} 4 p^{1}$ & $3 d^{2} 4 s^{1} 5 p^{1}$ & $3 d^{3} 5 p^{1}$ \\
\hline & & F.V. & C.C & F.V. & C.C & & \\
Eav & $121463^{\mathrm{f}}$ & $80,172(23)$ & & $52,332(15)$ & 50,535 & $119953^{\mathrm{f}}$ & $93789(22)$ \\
$\mathrm{F}^{2}$ (3d, 3d) & & $62,803(144)$ & $62,803^{\mathrm{a}}$ & $56,187(70)$ & 56,652 & $63419^{\mathrm{f}}$ & $57493^{\mathrm{f}}$ \\
$\mathrm{F}^{4}$ (3d, 3d) & & $39,639(227)$ & 39,299 & $33,419(159)$ & 35,212 & $39715^{\mathrm{f}}$ & $35775^{\mathrm{f}}$ \\
$\mathrm{F}^{2}$ (3d, np) & $14228^{\mathrm{f}}$ & $16,167(101)$ & 14,685 & $11,842(82)$ & 11,471 & $3150^{\mathrm{f}}$ & $3161^{\mathrm{f}}$ \\
$\mathrm{G}^{1}$ (3d, np) & $5002^{\mathrm{f}}$ & $6011(110)$ & 4855 & $5507(39)$ & 4928 & $3662^{\mathrm{f}}$ & $1173^{\mathrm{f}}$ \\
$\mathrm{G}^{3}$ (3d, np) & $4472^{\mathrm{f}}$ & $3697(267)$ & 5402 & $2239(129)$ & 3961 & $964^{\mathrm{f}}$ & $1028^{\mathrm{f}}$ \\
$\mathrm{G}^{1}$ (4s, np) & & $34,041(114)$ & 31,226 & & & $1054^{\mathrm{f}}$ & \\
$\mathrm{G}^{2}$ (3d, 4s) & & $7694(217)$ & 11,629 & & & $8668^{\mathrm{f}}$ & \\
$\zeta_{3 \mathrm{~d}}$ & $236^{\mathrm{f}}$ & $206(13)$ & 206 & $168(10)$ & 176 & $208^{\mathrm{f}}$ & $179^{\mathrm{f}}$ \\
$\zeta_{\text {np }}$ & $363^{\mathrm{f}}$ & $332(37)$ & 275 & $259(23)$ & 204 & $76^{\mathrm{f}}$ & $66^{\mathrm{f}}$ \\
\hline
\end{tabular}

F.V.: fitted value; C.C.: Cowan code; ${ }^{\text {a }}$ fixed to fitted value; ${ }^{\text {f }}$ fixed to weighted $a b$ initio value (C.C.).

Table 3. Fitted configuration interaction parameters.

\begin{tabular}{|c|c|c|}
\hline \multicolumn{3}{|c|}{ Values of main configuration interaction parameters in $\cdot \mathrm{cm}^{-1}$} \\
\hline $3 d 4 s^{2} 4 p-3 d^{2} 4 s 4 p$ & $\mathrm{R}^{1}(5 \mathrm{p} 5 \mathrm{~s}, 4 \mathrm{~d} 5 \mathrm{p})$ & $-3359^{f}$ \\
\hline & $\mathrm{R}^{2}(5 \mathrm{~s} 5 \mathrm{p}, 4 \mathrm{~d} 5 \mathrm{p})$ & $-3370^{f}$ \\
\hline \multirow[t]{2}{*}{$3 d 4 s^{2} 4 p-3 d^{3} 4 p$} & $\mathrm{R}^{2}(5 \mathrm{~s} 5 \mathrm{~s}, 4 \mathrm{~d} 4 \mathrm{~d})$ & $11412^{\mathrm{f}}$ \\
\hline & $\mathrm{R}^{2}(5 \mathrm{p} 5 \mathrm{~s}, 4 \mathrm{~d} 5 \mathrm{p})$ & $-6048(371)$ \\
\hline \multirow[t]{2}{*}{$3 d^{2} 4 s 4 p-3 d^{3} 4 p$} & $\mathrm{R}^{1}(5 \mathrm{~s} 5 \mathrm{p}, 4 \mathrm{~d} 5 \mathrm{p})$ & -9559 (208) \\
\hline & $\mathrm{R}^{2}(4 \mathrm{~d} 5 \mathrm{~s}, 4 \mathrm{~d} 4 \mathrm{~d})$ & -3554 (187) \\
\hline
\end{tabular}


Table 4. Predicted positions for missing experimental energy levels of the $3 d^{2} 4 s 4 p$ configuration up to $100,000 \mathrm{~cm}^{-1}$, resulting LS-percentage of the largest wave function component and the corresponding calculated Landé gJ-factor.

\begin{tabular}{|c|c|c|c|c|c|}
\hline Configuration & $\mathrm{J}$ value & Designation & Energy $\left(\mathrm{cm}^{-1}\right)$ & Composition LS (\%) & Calc. g $g_{J}$ \\
\hline \multicolumn{6}{|l|}{$3 d^{2} 4 s 4 p$} \\
\hline \multicolumn{6}{|c|}{${ }^{3} \mathrm{~F},{ }^{1} \mathrm{P} ;{ }^{3} \mathrm{D}$} \\
\hline & 1 & & 89143 & 56 & 0.504 \\
\hline & 2 & & 89363 & 55 & 1.166 \\
\hline & 3 & & 89680 & 53 & 1.334 \\
\hline \multicolumn{6}{|c|}{${ }^{3} \mathrm{~F},{ }^{1} \mathrm{P} ;{ }^{3} \mathrm{~F}$} \\
\hline & 2 & & 88643 & 60 & 0.668 \\
\hline & 3 & & 88864 & 55 & 1.064 \\
\hline & 4 & & 89141 & 53 & 1.235 \\
\hline \multicolumn{6}{|c|}{${ }^{3} \mathrm{~F},{ }^{1} \mathrm{P} ;{ }^{3} \mathrm{G}$} \\
\hline & 3 & & 88548 & 72 & 0.771 \\
\hline & 4 & & 88809 & 70 & 1.065 \\
\hline & 5 & & 89152 & 74 & 1.200 \\
\hline \multicolumn{6}{|c|}{${ }^{3} \mathrm{P},{ }^{3} \mathrm{P} ;{ }^{5} \mathrm{D}$} \\
\hline \multirow{2}{*}{\multicolumn{6}{|c|}{75172}} \\
\hline & & & & & \\
\hline \multirow{2}{*}{\multicolumn{6}{|c|}{${ }^{3} \mathrm{P},{ }^{3} \mathrm{P} ;{ }^{5} \mathrm{~S}$}} \\
\hline & & & & & \\
\hline & 2 & & 73804 & 97 & 1.998 \\
\hline \multicolumn{6}{|c|}{${ }^{1} \mathrm{D},{ }^{3} \mathrm{P} ;{ }^{3} \mathrm{P}$} \\
\hline \multirow{2}{*}{\multicolumn{6}{|c|}{${ }^{1} \mathrm{D},{ }^{1} \mathrm{D} ;{ }^{1} \mathrm{~F}$}} \\
\hline & & & & & \\
\hline & 3 & & 99800 & 76 & 1.016 \\
\hline \multicolumn{6}{|c|}{${ }^{3} \mathrm{~F},{ }^{3} \mathrm{P} ;{ }^{1} \mathrm{G}$} \\
\hline & 4 & & 73589 & 96 & 1.001 \\
\hline
\end{tabular}

we suggest to compare the ratio: $a_{4 s}^{10}\left(3 d^{3} 4 s\right)_{\text {Fit }} / a_{4 s}^{10}\left(3 d^{2} 4 s 4 p\right)_{\text {Fit }}=\frac{4519.76}{6014}=0.7725$ obtained by using fitted experimental HFS V II data (Table 5 of Part I of this work and Table 5 of part II) to the same ratio $a_{4 s}^{10}\left(3 d^{3} 4 s\right)_{a b \text { initio }} / a_{4 s}^{10}\left(3 d^{2} 4 s 4 p\right)_{a b \text { initio }}=\frac{43.09}{55.77}=0.7726$ using the computed data given in Table 6, obtained thanks to use of the pseudo relativistic Hartree-Fock code [21]. We can see that these two ratios are very similar to the fourth decimal place and we can conclude that the $a_{4 s}^{10}\left(3 d^{2} 4 s 4 p\right)_{\text {Fit }}$ value is very satisfactory. In Table 5, comparing the $a b$ initio and fitted HFS one-electron parameters for $d-, p$ - and s-electrons we have achieved good agreement further verifying the veracity of the fitting model. Moreover we confirm through our calculations the well-founded basis of the experimental data of Armstrong, Rosner and Holt [20], gathered in Table 7 where one can note that experimental values are close to calculated ones except for the level whose energy is equal to $54718 \mathrm{~cm}^{-1}$. 
Table 5. The fitted and calculated main HFS many-body parameters (in MHz). The uncertainties given in parentheses are the standard deviations. Radial integrals are computed by means of the Cowan code.

\begin{tabular}{|c|c|c|}
\hline Configuration & $3 d^{2} 4 s 4 p$ & $3 d^{3} 4 p$ \\
\hline$\left\langle\mathrm{r}^{-3}\right\rangle_{4 \mathrm{~d}}$ (a.u.) & 3.040 & 2.646 \\
\hline Ab-initio & 206 & 176 \\
\hline b3d (cint) & 206.18 (12.79) & $167.71(10.38)$ \\
\hline$a_{4 d}^{01} \quad \mathrm{cal}(\mathrm{MHz})$ & 427.04 & 353.88 \\
\hline$a_{4 d}^{01} \quad$ (Fit) (MHz) & 426.17 (1.47) & 367.36 (1.26) \\
\hline$\left\langle\mathrm{r}^{-3}\right\rangle_{\mathrm{np}}$ (a.u.) & 2.378 & 1.761 \\
\hline Ab-initio & 275 & 204 \\
\hline$\zeta_{4 p}\left(\mathrm{~cm}^{-1}\right) \quad$ Fit & 331.79 (37.16) & $258.91(22.72)$ \\
\hline$a_{4 p}^{01} \quad \operatorname{cal}(\mathrm{MHz})$ & 402.68 & 313.69 \\
\hline$a_{4 p}^{01} \quad$ (Fit) (MHz) & 394.23 (7.30) & 292.13 \\
\hline$a_{4 s}^{10}($ Fit) $(\mathrm{MHz})$ & $6014(246)$ & \\
\hline$a_{4 s}^{10} \quad$ cal $(\mathrm{MHz})$ & 5851.14 & \\
\hline
\end{tabular}

Table 6. Pseudo-relativistic Hartree-Fock estimates of $4 \pi|\Psi(0)|^{2}$ (in units of $\mathrm{a}_{0}{ }^{-3}$ ) for configurations of interest here, using the PSUHFR code [21].

\begin{tabular}{ccc}
\hline Configuration & $3 d^{2} 4 s^{1} 4 p^{1}$ & $3 d^{3} 4 s^{1}$ \\
\hline $1 \mathrm{~s}$ & 51,903 & 51,906 \\
$2 \mathrm{~s}$ & 4638 & 4638 \\
$3 \mathrm{~s}$ & 622 & 606 \\
$4 \mathrm{~s}$ & 55.77 & 43.09 \\
Total $^{\mathrm{a}}$ s-electron density & 114,380 & 114,345 \\
\hline
\end{tabular}

${ }^{\mathrm{a}}$ Totals are contribution from all s-orbitals weighted by their occupation numbers.

Table 7. Predicted HFS A constants of ${ }^{51}$ V II (in MHz), compared with those obtained experimentally by Armstrong, Rosner and Holt [20].

\begin{tabular}{cccc}
\hline Level $\left(\mathrm{cm}^{-1}\right)$ & $\mathrm{J}$ value & $\mathrm{A}(\mathrm{MHz})_{\text {Experimental }}[20]$ & $\mathrm{A}(\mathrm{MHz})_{\text {Calculated }}$ This work \\
36489 & 1 & 715.84 & 701.18 \\
36955 & 1 & 827.39 & 816.95 \\
52604 & 1 & 532.12 & 550.05 \\
52804 & 1 & 496.9 & 490.26 \\
53751 & 1 & 432.83 & 440.04 \\
54718 & 1 & 38.93 & 67.84 \\
55663 & 1 & -317.77 & -259.60 \\
36673 & 2 & 239.52 & 238.51 \\
37041 & 2 & 300.37 & 307.70 \\
40002 & 2 & 609.63 & 613.78 \\
\hline
\end{tabular}




\begin{tabular}{|c|c|c|c|}
\hline \multicolumn{4}{|l|}{ Continued } \\
\hline 52246 & 2 & 520.47 & 521.79 \\
\hline 52700 & 2 & 270.37 & 258.59 \\
\hline 53869 & 2 & 222.87 & 232.10 \\
\hline 54716 & 2 & 131.00 & 138.93 \\
\hline 57343 & 2 & 342.90 & 343.33 \\
\hline 37205 & 3 & 160.22 & 177.29 \\
\hline 39234 & 3 & 501.86 & 498.12 \\
\hline 40196 & 3 & 301.13 & 301.48 \\
\hline 49211 & 3 & 400.45 & 425.61 \\
\hline 49568 & 3 & 326.76 & 327.53 \\
\hline 52392 & 3 & 326.62 & 315.08 \\
\hline 52767 & 3 & 141.03 & 128.18 \\
\hline 53927 & 3 & 233.25 & 236.59 \\
\hline 66304 & 3 & 397.45 & 429.20 \\
\hline 37531 & 4 & 85.61 & 98.32 \\
\hline 39404 & 4 & 304.11 & 304.40 \\
\hline 40430 & 4 & 178.23 & 174.53 \\
\hline 52657 & 4 & 239.99 & 227.86 \\
\hline 62176 & 4 & 351.28 or -276.67 & 339.91 \\
\hline 37352 & 5 & 161.02 & 165.45 \\
\hline 39613 & 5 & 213.51 & 216.29 \\
\hline
\end{tabular}

\section{Conclusion}

We studied the spectrum of V II which permits to point out the incorrect positions of three triplets. We give refined fine structure parameters and leading eigenvectors percentages of levels and for the first time the calculated magnetic Landé gi-factors, which are very useful for missing level assignments. Taking advantage of recent experimental work on hyperfine structure of this ion [20] we were also able to determine for the first time the predominant single-electron HFS parameter values, confirmed using $a b$ initio calculations. It would be interesting to extend this study experimentally to the missing levels of $3 d^{2} 4 s 4 p$ to compare with our predicted positions. Further experimental work on the unknown levels of $3 d^{3} 4 p, 3 d^{3} 5 p, 3 d 4 s^{2} 4 p, 4 d 5 s^{2} 5 p$ and $3 d^{2} 4 s 5 p$ configurations would also be useful, since the situation is already formulated for future investigations of all existing levels of this basis.

\section{References}

[1] Meggers, W.F. and Moore, C.E. (1940) Journal of Research of the National Bureau of Standards (US), 25, 83.

[2] Velasco, R. and Gullon, N. (1968) Optica Pura y Aplicada, 1, 93.

[3] Iglesias, L. (1977) Optica Pura y Aplicada, 10, 267.

[4] Sugar, J. and Corliss, C.H. (1978) Journal of Physical and Chemical Reference Data, 7, 1207.

[5] Roth, C. (1969) Journal of Research of the National Bureau of Standards, 73A, 125. http://dx.doi.org/10.6028/ires.073A.012

[6] Roth, C. (1969) Journal of Research of the National Bureau of Standards, 73A, 159. 
http://dx.doi.org/10.6028/jres.073A.013

[7] Iglesias, L. and Cabeza, M.I. (1988) Optica Pura y Aplicada, 21, 139.

[8] Bouazza, S. (2012) Physica Scripta, 86, 015302. http://dx.doi.org/10.1088/0031-8949/86/01/015302

[9] Bouazza, S. (2013) Physica Scripta, 87, 035303. http://dx.doi.org/10.1088/0031-8949/87/03/035303

[10] Dembczynski, J. and Stachowska, E. (1991) Physica Scripta, 43, 248. http://dx.doi.org/10.1088/0031-8949/43/3/006

[11] Bouazza, S., Behrens, H.O., Fienhold, M., Dembczynski, J. and Guthöhrlein, G.H. (1999) European Physical Journal D, 6, 311-317. http://dx.doi.org/10.1007/s100530050314

[12] Bouazza, S., Hannaford, P. and Wilson, M. (2003) Journal of Physics B, 36, 1537. http://dx.doi.org/10.1088/0953-4075/36/8/308

[13] Armstrong, L. (1971) Theory of the Hyperfine Structure of Free Atoms. Wiley, New York.

[14] Lingren, I. and Morisson, I. (1982) Many-Body Theory. Springer, Berlin. http://dx.doi.org/10.1007/978-3-642-96614-9

[15] Lingren, I. and Rosen, A. (1970) Case Studies At. Physics, 4, 97.

[16] Armstrong, L. and Feneuille, S. (1974) Advances in Atomic and Molecular Physics, 10, 1-52. http://dx.doi.org/10.1016/S0065-2199(08)60346-2

[17] Armstrong, L. and Feneuille, S. (1968) Physical Review, 173, 58. http://dx.doi.org/10.1103/PhysRev.173.58

[18] Cowan, R.D. (1981) The Theory of Atomic Structure and Spectra. University of California Press, Berkeley.

[19] Bouazza, S., Dembczynski, J., Stachowska, E., Szawiola, G. and Ruczkowski, J. (1998) European Physical Journal D, 4, 39.

[20] Armstrong, N.M.R., Rosner, S.D. and Holt, R.A. (2011) Physica Scripta, 84, 055301. http://dx.doi.org/10.1088/0031-8949/84/05/055301

[21] Wilson, M. (1978) Physica C, 95, 129. http://dx.doi.org/10.1016/0378-4363(78)90019-0 